Original Article

\title{
Modified feedback-control task for examining the relationship between sense of agency and motor control: a pilot study
}

\author{
Yu Miyawaki, RPT, MS ${ }^{1 *}$, TAKeshi Otani, RPT ${ }^{2)}$, Shu Morioka, RPT, PhD ${ }^{1,3)}$ \\ 1) Department of Neurorehabilitation, Graduate School of Health Science, Kio University: \\ 4-2-2 Umami-naka, Koryo-cho, Kitakaturagi-gun, Nara 635-0832, Japan \\ 2) Department of Rehabilitation, Ishikawa Hospital, Japan \\ 3) Neurorehabilitation Research Center, Kio University, Japan
}

\begin{abstract}
Purpose] A sense of agency and feedback control may be related when the sensory feedback is attributed to the self; however, the relationship between sense of agency and movement disorders remains unclear. Although a feedback-control task might enable the examination of this relationship, it may be difficult for patients with movement disorders to complete this task. The present study modified the feedback-control task for future clinical research. [Participants and Methods] Twenty-four healthy adults participated in the study. The basic procedure followed that of a previous study in which participants traced a target line while receiving visual feedback of their actual or fake movement. The task was modified to reduce the width of the movement area, change the shape of the line from sinusoidal to horizontal, and reduce the number of trials from 45 to 15. [Results] When participants received the visual feedback of their actual movement, the movement error significantly decreased, whereas when participants received the fake movement that represented pre-recordings of their previous own movements, the movement error significantly increased. [Conclusion] The results partially agreed with those of the previous study. This modified task might help in examining the relationship between sense of agency and movement disorders in terms of motor control.

Key words: Sense of agency, Motor control, Forward model
\end{abstract}

(This article was submitted Dec. 10, 2018, and was accepted Feb. 1, 2019)

\section{INTRODUCTION}

The sense of agency (the experience of controlling one's own action) ${ }^{1,2)}$ arises from attributing the sensory consequences to the self. This experience plays an important role in motor control. Some studies indicated that motor control can be confused by the illusory self-attribution of externally generated sensations ${ }^{3,4)}$. This confusion was due to utilizing the visual feedback of others' movement as that of self-movement to realize feedback control. Therefore, these findings suggest that we can correct our own movement by attributing self-generated sensations to ourselves. The sense of agency (i.e., selfattribution) may have the function of "the mediator between sensory input and motor output" (p. 127) $)^{4}$, based on the system of self-other sensory attribution.

Regarding this system, some studies have suggested that sense of agency may depend on the consistency between predicted sensations and actual sensory feedback ${ }^{5-7)}$. Specifically, according to the forward model in the brain, the sensory prediction based on an efference copy of motor command is compared with actual sensory feedback ${ }^{8,9)}$. If actual sensory feedback matches the prediction, the sensation will be attributed to the self ${ }^{5)}$. In this viewpoint, Asai examined the dynamic relationship between self-other attribution and feedback control ${ }^{4}$ ). The findings indicated that when participants performed a

*Corresponding author. Yu Miyawaki (E-mail: yu.miyawaki.reha1@gmail.com)

(C2019 The Society of Physical Therapy Science. Published by IPEC Inc.

(c) (i) $\odot$ This is an open-access article distributed under the terms of the Creative Commons Attribution Non-Commercial No Derivatives cc) 
sinusoidal movement while receiving visual feedback of a fake movement that was spatiotemporally similar to their actual movement, they made illusory self-attribution of the fake movement and tried to control its movement ${ }^{4}$. This misattribution depended on the level of difficulty in detecting the error between their actual movement and the visual feedback ${ }^{4}$. Therefore, self-other sensory attribution may be linked with the sensorimotor system in the brain ${ }^{4,10)}$.

Although some studies have examined the relationship between self-other sensory attribution and motor $\operatorname{control}^{3,4,11)}$, in terms of movement disorders, the relationship between sense of agency and these disorders remains unclear. In particular, as self-other sensory attribution may be influenced by the sensorimotor system ${ }^{4}, 10$ ) , post-stroke patients with damage to brain regions involved in the sensorimotor system might present an altered sense of agency. Given that sense of agency exerts an important function in motor control ${ }^{3,4,11)}$, clinical studies investigating the relationship between sense of agency and movement disorders are needed.

Asai's experimental paradigm ${ }^{4}$ may facilitate the examination of this relationship. However, in terms of clinical practice, this seminal study ${ }^{4}$ could present difficulties for patients with movement disorders, such as post-stroke patients; the feedback-control task (sinusoidal reaching movement with its visual feedback) may be too complex for post-stroke patients, whose arm movements are often accompanied by synkinetic contractions ${ }^{12}$ ) because these patients would be required to execute multi-joint, independent movements of their upper limb. Moreover, this task consists of numerous trials (45 trials, each consisting of the sinusoidal movement lasting $10 \mathrm{~s}$ ), which may lead to physical and mental fatigue in the patients. Therefore, simplifying the task and reducing the number of trials would be key in facilitating these clinical studies. The present study aimed to simplify the feedback-control task for future clinical studies by examining whether the modified task resulted in findings consistent with those of Asai's study ${ }^{4}$.

\section{PARTICIPANTS AND METHODS}

Twenty-four healthy adults consisting of 11 males and 13 females (mean age $=24.6 \pm 1.8$ years; mean height $=163.9 \pm$ $8.7 \mathrm{~cm}$; mean weight $=57.7 \pm 11.7 \mathrm{~kg}$ ) participated in the study. All participants were randomly assigned to the own-fake group $^{4)}$ or the others'-fake group ${ }^{4}$ with 12 participants in each, resulting in five males and seven females (mean age $=24.3 \pm$ 1.7 years; mean height $=162.4 \pm 9.2 \mathrm{~cm}$; mean weight $=56.0 \pm 13.1 \mathrm{~kg}$ ) in the own-fake group and six males and six females (mean age $=24.9 \pm 2.0$ years; mean height $=165.4 \pm 8.4 \mathrm{~cm}$; mean weight $=59.4 \pm 10.4 \mathrm{~kg}$ ) in the others'-fake group. Each participant provided written informed consent. This study was approved by the ethics committee of the Jinjukai Ishikawa Hospital (2018-1) and conducted in accordance with the ethical standards of the 1964 Declaration of Helsinki.

The basic procedure followed that of Asai's study, in which participants traced a target line (sine wave composed of 5 cycles) and received its visual feedback (circle cursor, $9 \mathrm{~mm}$ in diameter) ${ }^{4}$. To simplify the task, we made three modifications. First, to make the range of movement smaller, we used the monitor of a laptop computer (plotting area: $263 \times 163 \mathrm{~mm}$, CF-SV7TBAQP, Panasonic), instead of the monitor (plotting area: $488 \times 305 \mathrm{~mm}$ ) used in Asai's study). The monitor, which displayed the target line and the visual feedback, was set $20 \mathrm{~cm}$ vertically above a digitizing tablet (Intuos Pro Large PTH-851/K, Wacom) in accordance with the setup in Asai's study ${ }^{4}$. Moreover, the size of the input area of the digitizer was set to be similar to that of the plotting area of the monitor. The refresh rate of the monitor was $60 \mathrm{~Hz}$. Second, the target line was changed from the sine wave to a straight line and was displayed horizontally on the middle of the monitor. Therefore, participants were required to perform horizontal movements (i.e., horizontally manipulate the pen) on the digitizer to trace the target line on the monitor as accurately as possible. This task was adopted in the present study because the horizontal movement could be executed with a single-joint shoulder movement: it is our belief that post-stroke patients capable of independent shoulder movement will be able to perform this task in future studies. During the preparation time of $5 \mathrm{~s}$, participants placed the pen at the start position (left end of the target line). When the computer started to count up (i.e., at zero), participants moved the pen towards the right end of the target line from the start position. Participants performed 4 cycles of the horizontal movements so that the timing of reaching the end (left or right) of the target line matched to the counting from 1 to $8 \mathrm{~s}$. The movement error, which was defined as the vertical distance between the pen position and the target line, was measured as the index of the motor performance ${ }^{4)}$. Third, the number of trials in the main experiment was reduced from the 45 in Asai's ${ }^{4)}$ study to 15 . Moreover, the number of trials in the practice session was reduced from $40^{4)}$ to 15.

The experimental paradigm for examining the relationship between self-other sensory attribution and motor control followed that of Asai's study in which there were three conditions with respect to the visual feedback of participants' pen movements: SELF, FAKE, and NO visual feedback condition ${ }^{4}$. Each condition consisted of 5 trials in a random order. In the SELF condition, participants received the visual feedback of their actual movement as the cursor movement, and then participants were required to control the cursor. In the FAKE condition, participants received the different fake movements of the cursor in each group. For the own-fake group, the fake movements represented the participants' own movements that had been secretly recorded in the practice session. Since the first 5 trials in the practice session were not used as the fake movements, the fake movements were randomly chosen from data of the last 10 trials. For the others'-fake group, the fake movements represented others' movements that were randomly chosen from the data sets of the other 14 participants' movements. Participants were required to ignore the fake movement and trace the target line by using their proprioception. After participants started to move, the cursor was masked for first and last $0.5 \mathrm{~s}$ in order to make it impossible to distinguish between participants' actual and fake movements by the timing of starting and finishing the movement. 
Table 1. Comparison of the movement error between conditions in the own-fake and others'-fake groups

\begin{tabular}{|c|c|c|c|c|c|c|c|c|}
\hline & \multicolumn{4}{|c|}{$\begin{array}{l}\text { Own-fake group } \\
(\mathrm{n}=12)\end{array}$} & \multicolumn{4}{|c|}{$\begin{array}{l}\text { Others'-fake group } \\
\qquad(\mathrm{n}=12)\end{array}$} \\
\hline & Cycle 1 & Cycle 2 & Cycle 3 & Cycle 4 & Cycle 1 & Cycle 2 & Cycle 3 & Cycle 4 \\
\hline SELF (pix) & $49.9 \pm 19.2^{*}$ & $38.3 \pm 12.0^{*}$ & $33.9 \pm 9.2^{*}$ & $33.5 \pm 6.0^{*}$ & $41.8 \pm 13.2$ & $40.3 \pm 16.7^{*}$ & $31.8 \pm 8.9^{*}$ & $28.0 \pm 8.3^{*}$ \\
\hline FAKE (pix) & $58.1 \pm 23.6$ & $69.2 \pm 29.5$ & $77.6 \pm 33.8$ & $84.1 \pm 36.1^{\dagger}$ & $45.1 \pm 14.6$ & $55.5 \pm 27.5$ & $52.6 \pm 30.2$ & $56.1 \pm 32.7$ \\
\hline NO (pix) & $62.3 \pm 29.9$ & $64.2 \pm 33.4$ & $65.6 \pm 29.2$ & $67.8 \pm 31.5$ & $48.3 \pm 14.3$ & $51.1 \pm 20.1$ & $50.5 \pm 18.1$ & $53.0 \pm 14.5$ \\
\hline
\end{tabular}

Values are presented as mean \pm standard deviation $(1 \mathrm{pix}=0.14 \mathrm{~mm})$.

*Significant differences between SELF and FAKE and between SELF and NO $(\mathrm{p}<0.05)$.

†Significant difference between FAKE and NO $(\mathrm{p}=0.047)$.

The trajectory of the fake movements for the own-fake group had spatiotemporal characteristics similar to those of the participant's actual movements ${ }^{4}$. Therefore, these fake movements exerted greater difficulty for participants in detecting the prediction error ${ }^{13)}$ between actual and fake movements than those in the others'-fake group. According to Asai's study ${ }^{4)}$, if participants judged that the fake movement represented the visual feedback of their actual movement (i.e., illusory selfattribution), they would try to correct the cursor movement when it deviated from the target line. In this instance, since participants cannot control the fake movement, its movement error would increase due to the illusory feedback control. Conversely, if participants ignored the cursor movement, its movement error would be similar to that of the NO visual feedback condition in which participants traced the target line without the cursor (i.e., the NO condition was defined as the baseline of the SELF and FAKE conditions). Therefore, participants were required to correctly make self-other attribution of the cursor to accurately control their actual movement.

During each trial, participants were required to judge whether the cursor movement was their own or fake movement. After each trial, participants reported their subjective judgment of self-other with respect to the cursor by rating on a scale ranging from 1 (completely others' movement) to 9 (completely self-movement). This subjective judgment of self-other was reported by pressing the corresponding key for each. The 9-point scale was adopted in the present study to examine the subjective judgment of self-other in more detail ${ }^{14)}$, replacing the two-alternative forced choice task adopted in Asai's study ${ }^{4}$. The visual or auditory stimuli were programmed using Hot Soup Processor 3.4 (Onion Software).

The motor performance was analyzed by calculating the mean of the movement error for each cycle. Then, for each group, a two-way analysis of variance (ANOVA) was conducted with condition (SELF, FAKE, and NO visual feedback) as a withinparticipants independent variable, and cycle (4 cycles) as a within-participants independent variable, and with movement error as the dependent variable. Concerning the analysis of the judgment of self-other, to quantify the subjective misattribution with respect to the cursor, we subtracted the mean value of the 9-point scale from 9 (completely self-movement) for the SELF condition, and 1 (completely others' movement) from the mean value for the FAKE condition. A two-way ANOVA was conducted with group (own-fake and others'-fake) as the between-participants independent variable, condition (SELF and FAKE) as the within-participants independent variable, and subjective misattribution as the dependent variable. The modified Bonferroni method was used for multiple comparisons. The significant level was set at $\mathrm{p}<0.05$. All analyses were conducted using R version 3.3.2 (R Foundation for Statistical Computing, Vienna, Austria).

\section{RESULTS}

In motor performance (Table 1), for the own-fake group, a significant interaction between condition and cycle was observed $(F=11.33, \mathrm{p}<0.001)$. Concerning the differences among conditions, simple main effects were significant in all cycles $(\mathrm{p}<0.006)$. Post hoc analysis indicated that the movement error of the SELF condition was significantly smaller than those in the FAKE and NO conditions in all cycles $(\mathrm{p}<0.05)$, and the movement error in the FAKE condition was significantly larger than that in the NO condition in cycle $4(\mathrm{p}=0.047)$. Regarding the differences among cycles, simple main effects were significant in the SELF ( $p=0.019)$ and FAKE ( $\mathrm{p}=0.008)$ conditions. Post hoc analysis indicated no significant differences among cycles in the SELF condition ( $>0.05)$ and significant differences among the other cycles $(\mathrm{p}<0.05)$, excluding between cycles 1 and 2 ( $\mathrm{p}=0.059)$, in the FAKE condition.

For the others'-fake group, a significant interaction between condition and cycle was observed $(F=4.55, \mathrm{p}<0.023)$. Simple main effects of the differences among conditions were significant in cycles $2-4(\mathrm{p}<0.05)$. Post hoc analysis indicated that the movement error in the SELF condition was significantly smaller than those in the FAKE and NO conditions in cycles 2-4 $(p<0.05)$, and there were no significant differences between the FAKE and NO conditions in all cycles $(p>0.05)$. Concerning the differences among cycles, simple main effects were significant in the SELF $(p<0.001)$ condition. Post hoc analysis indicated significant differences between cycles 1 and $4(\mathrm{p}<0.001)$ and between 2 and $4(\mathrm{p}=0.009)$ in the SELF condition.

In the judgment of self-other (Table 2$)$, significant main effects of group $(F=5.83, \mathrm{p}=0.024)$ and condition $(F=5.87$, $\mathrm{p}=0.024$ ) were observed. 
Table 2. Comparison of the subjective misattribution between conditions in the own-fake and others'-fake groups*

\begin{tabular}{ccc}
\hline & $\begin{array}{c}\text { Own-fake group } \\
(\mathrm{n}=12)\end{array}$ & $\begin{array}{c}\text { Others'-fake group } \\
(\mathrm{n}=12)\end{array}$ \\
\hline SELF $^{\dagger}$ & $0.8 \pm 1.0$ & $0.4 \pm 0.6$ \\
FAKE $^{\ddagger}$ & $1.9 \pm 1.5$ & $0.6 \pm 1.0$ \\
\hline
\end{tabular}

Values are presented as mean \pm standard deviation.

*Incorrect responses (misattributions) on the subjective judgment of self-other based on a 9-point scale.

†In the SELF condition, "misattribution" refers to other-attribution of self-movement.

\#n the FAKE condition, "misattribution" refers to self-attribution of fake movement.

\section{DISCUSSION}

For both groups, the movement error in the SELF condition was significantly smaller than those in the FAKE and NO conditions. Because these results provide evidence that participants were able to show feedback control, it seems reasonable to utilize this modified task as a feedback-control task. Importantly, for the own-fake group, the movement error in the FAKE condition was significantly larger than in the NO condition at cycle 4, although there was no significant difference between the FAKE and NO conditions for the others'-fake group. These results are consistent with the findings of Asai's study ${ }^{4}$. Therefore, the increased movement error may have been due to the illusory self-attribution of the fake movement (i.e., illusory feedback control). The modified task might have the ability to examine the dynamic relationship between self-other attribution and feedback control.

The results of the present study include two main differences from those of Asai's study ${ }^{4}$. First, regarding the NO condition, the current study showed no significant differences among cycles, whereas Asai's study indicated significant increases of movement errors among cycles. The difference between these results may be explained by the specific tasks chosen (horizontal or sinusoidal ${ }^{4}$ movement). Because it is conceivable that performing the horizontal movement is generally easier than performing the sinusoidal movement, the modified task might have enabled participants to make stable movements through all cycles even without the visual feedback. This explanation is supported by the fact that the overall movement errors in the present study were smaller than those of Asai's study ${ }^{4}$. Taken together, these findings suggest that the difficulty level of the feedback-control task was reduced by modifying the task, as we expected.

Second, in the judgements of self-other, the results indicated significant main effects of group and condition (i.e., significant misattributions in the own-fake group and the FAKE condition), whereas Asai's study only indicated a significant main effect of condition (i.e., significant misattribution in the FAKE condition) ${ }^{4}$ ). Some studies have claimed that the explicit judgement of agency (i.e., subjective judgement of self-other) is sensitive to a cognitive bias ${ }^{15,16)}$. Because participants for the own-fake group experienced greater difficulty in distinguishing between their actual and fake movements in the FAKE condition, they might have been affected by the cognitive bias such as the doubt based on their experienced difficulty, even in the SELF condition. Moreover, the present study used a 9-point scale to examine the subjective misattribution in more detail $^{14)}$, instead of the two-alternative forced choice task ${ }^{4}$. Consequently, the 9-point scale might have detected the influence of the cognitive bias on the self-other judgement for the own-fake group. As mentioned in Asai's study, the subjective judgment of self-other is limited in that it cannot examine the time course of the explicit agency ${ }^{4}$. In addition to this limitation, the validity of the 9-point scale used in this experimental paradigm should be studied further.

Several limitations concerning the modified task should be considered. While, this task may display strengths in its simplicity for use in clinical practice, it may also contain methodological problems in that the visual feedback might not be necessary for tracing the straight line due to participants performing linear movements particularly accurately. According to the results of this study, the visual feedback was utilized for correcting the horizontal movements (i.e., significant differences between the SELF and NO conditions for both groups). Because the training beyond simple familiarization with the task could induce mastery of the horizontal movement skill, reducing the number of trials in the practice session might have contributed to these results. However, it remains unclear whether this number of trials is appropriate for patients with movement disorders to become familiar with the task. Further studies investigating the appropriate number of trials for the patients should be conducted. Moreover, the characteristics of participants in the horizontal movement would be more similar compared with the characteristics in the sinusoidal movement ${ }^{4}$. This similarity could make the distinction between self- and fake movements more difficult in post-stroke patients with an impaired sensorimotor system although the results of the present study with healthy adults were consistent with those of Asai's study ${ }^{4}$. By resolving these limitations, the modified task may be useful in the examination of the relationship between sense of agency and motor control in patients with movement disorders.

In conclusion, using the modified feedback-control task, this study provided evidence in partial agreement with the find- 
ings of Asai's study ${ }^{4}$ that examined the dynamic relationship between self-other sensory attribution and feedback control. In terms of motor control, this modified task might aid in the examination of the relationship between sense of agency and motor control in patients with movement disorders, such as post-stroke patients.

\section{Presentation at a conference}

A part of our study was presented in a conference (The 23rd Congress of Japanese Society of Physical Therapy Fundamentals: http://23thjptakiso.com/data/program_abstracts.pdf).

\section{Conflict of interest}

The authors declare no competing interest.

\section{REFERENCES}

1) Gallagher I: Philosophical conceptions of the self: implications for cognitive science. Trends Cogn Sci, 2000, 4: 14-21. [Medline] [CrossRef] Haggard P, Chambon V: Sense of agency. Curr Biol, 2012, 22: R390-R392. [Medline] [CrossRef]

Nielsen TI: Volition: a new experimental approach. Scand J Psychol, 1963, 4: 225-230. [CrossRef]

4) Asai T: Feedback control of one's own action: self-other sensory attribution in motor control. Conscious Cogn, 2015, 38: 118-129. [Medline] [CrossRef]

5) Sato A, Yasuda A: Illusion of sense of self-agency: discrepancy between the predicted and actual sensory consequences of actions modulates the sense of selfagency, but not the sense of self-ownership. Cognition, 2005, 94: 241-255. [Medline] [CrossRef]

6) Frith CD, Blakemore SJ, Wolpert DM: Abnormalities in the awareness and control of action. Philos Trans R Soc Lond B Biol Sci, 2000, 355: 1771-1788. [Medline] [CrossRef]

7) Blakemore SJ, Wolpert DM, Frith CD: Abnormalities in the awareness of action. Trends Cogn Sci, 2002, 6: 237-242. [Medline] [CrossRef]

8) Wolpert DM, Miall RC, Kawato M: Internal models in the cerebellum. Trends Cogn Sci, 1998, 2: 338-347. [Medline] [CrossRef]

9) Wolpert DM, Ghahramani Z, Jordan MI: An internal model for sensorimotor integration. Science, 1995, 269: 1880-1882. [Medline] [CrossRef]

10) Weiss C, Tsakiris M, Haggard P, et al.: Agency in the sensorimotor system and its relation to explicit action awareness. Neuropsychologia, $2014,52: 82-92$. [Medline] [CrossRef]

11) Toyomura A, Omori T: Auditory feedback control during a sentence-reading task: effect of other's voice. Acoust Sci Technol, 2005, 26: 358-361. [CrossRef]

12) Mazevet D, Meunier S, Pradat-Diehl P, et al.: Changes in propriospinally mediated excitation of upper limb motoneurons in stroke patients. Brain, 2003, 126: 988-1000. [Medline] [CrossRef]

13) Wolpert DM, Flanagan JR: Motor prediction. Curr Biol, 2001, 11: R729-R732. [Medline] [CrossRef]

14) Asai T: Self is "other", other is "self”: poor self-other discriminability explains schizotypal twisted agency judgment. Psychiatry Res, 2016, 246: 593-600. [Medline] [CrossRef]

15) Haggard P: Sense of agency in the human brain. Nat Rev Neurosci, 2017, 18: 196-207. [Medline] [CrossRef]

16) Wegner DM, Wheatley T: Apparent mental causation. Sources of the experience of will. Am Psychol, 1999, 54: 480-492. [Medline] [CrossRef] 